Research Paper

\title{
Impact of Prognostic Nutritional Index on Overall Survival for Patients with Metastatic Urothelial Carcinoma
}

\author{
Meng-Che Hsieh1, Kun-Ming Rau1, Po-Hui Chiang³, Ming-Tse Sung2, Jui Lan², Hao-Lun Luo ${ }^{3}$, Chun-Chieh \\ Huang ${ }^{4}$, Cheng-Hua Huang ${ }^{1}$, Harvey Yu-Li Su ${ }^{1,5}$ \\ 1. Division of Hematology Oncology, Department of Internal Medicine, Kaohsiung Chang Gung Memorial Hospital and Chang Gung University, College of \\ Medicine, Taiwan \\ 2. Department of Pathology, Kaohsiung Chang Gung Memorial Hospital and Chang Gung University, College of Medicine, Taiwan \\ 3. Department of Urology, Kaohsiung Chang Gung Memorial Hospital and Chang Gung University, College of Medicine, Taiwan \\ 4. Department of Radiation Oncology, Kaohsiung Chang Gung Memorial Hospital and Chang Gung University, College of Medicine, Taiwan \\ 5. Clinical Trial Center, Kaohsiung Chang Gung Memorial Hospital, Taiwan
}

$\square$ Corresponding author: Division of Hematology-Oncology, Department of Internal Medicine, Kaohsiung Chang Gung Memorial Hospital and Chang Gung University, College of Medicine, Taiwan. Address: No.123, Dapi Rd., Niaosong Dist., Kaohsiung City 833, Taiwan (R.O.C.). Phone: +886-7-7317123 ext. 8303. Fax: +886-7-7322402. E-mail: yolisu@mac.com

(c) Ivyspring International Publisher. This is an open access article distributed under the terms of the Creative Commons Attribution (CC BY-NC) license (https://creativecommons.org/licenses/by-nc/4.0/). See http://ivyspring.com/terms for full terms and conditions.

Received: 2018.01.21; Accepted: 2018.04.17; Published: 2018.06.14

\begin{abstract}
Background: Prognostic nutritional index (PNI) has been studied in various types of cancer which is significantly correlated with prognosis. The study aims to investigate the predictive role of PNI in patients with metastatic urothelial carcinoma (mUC) treated with systemic chemotherapy.

Methods: We retrospectively reviewed 141 patients with mUC who received systemic chemotherapy. PNI was calculated as $10 \times$ serum albumin concentration $(\mathrm{g} / \mathrm{dL})+0.005 \times$ lymphocyte count (number/mm ${ }^{2}$ ). The optimal cut-off value for PNI was estimated by using receiver operating curve analysis. Independent factors associated with progression-free survival (PFS) and overall survival (OS) were determined by Cox proportional regression models.

Results: The recommended cut-off value for PNI was 40. Patients with a low PNI had more visceral metastases $(p<0.0001)$, leukocytosis $(p=0.006)$, and anemia $(p<0.0001)$. On univariate analysis, patients with a low PNI had poor OS than those with a high PNI $(p<0.0001)$. The multivariate analysis showed PNI was an independent factor to predict OS ( $P=0.001)$.

Conclusions: Our study showed PNI is an independent prognostic factor in patients with mUC. Our work is clinically useful for anticipation of outcomes, risks stratification in clinical studies as well as patients counseling.
\end{abstract}

Key words: prognostic nutritional index, metastatic urothelial carcinoma, chemotherapy, overall survival, prognosis

\section{Introduction}

Urothelial carcinoma (UC) is the leading cancer of genitourinary tract.[1] Although great advances in anti-cancer treatment in recent years had achieved, the prognosis of metastatic urothelial carcinoma (mUC) remains poor and unsatisfied.[2] Cisplatin-based chemotherapy is still the standard treatment for mUC.[3] In general, the median overall survival (OS) on cisplatin combination chemotherapy is 14 months. Based on previous post-hoc analysis of clinical trials, some prognostic factors were identified to predict outcomes. For example, age,[4] performance status, [5] presence of liver metastasis,[6] baseline renal function,[7] number of metastatic site,[8] histopathologic variant[9] and neutrophil-to- 
lymphocyte ratio (NLR) were significantly associated with overall survival. Therefore, it is clinically valuable to explore more accurate and representative risk factors to improve patient's survival and provide information when consulting.

For decades, the nutritional and inflammatory status has been demonstrated to be associated with survival in patients with cancer.[10-13] Notably, the prognostic nutritional index (PNI) is one of the most reliably predictive models of cancer patients by calculating serum albumin level and total lymphocyte counts. PNI was firstly proposed by Onodera et al. in 1984 to assess the perioperative immunonutritional status and surgical risks in patients underwent gastrointestinal surgery.[14] However, increasing numbers of studies demonstrated that PNI has been applied as a prognostic factor in patients with various metastatic malignancies.[15-18] With regard to urothelial carcinoma, the prognostic role of PNI is not fully evaluated. Therefore, this retrospective study aims to investigate the correlation between clinicopathologic factors and PNI, and to verify the survival impact of PNI on patients with mUC.

\section{Patients and Methods}

\section{Patients, treatment and primary outcome}

The study was approved by the Chang Gung Medical Foundation Institutional Review Board and the informed consent requirement was also waived because it was a retrospective study. All methods used in this study were performed in accordance with the relevant guidelines and regulations of the local ethical committee. We retrospectively analyzed 383 consecutive patients with metastatic UC from January 1997 to December 2015 in Kaohsiung Chang Gung Memorial Hospital, Taiwan. Database variables were recorded from medical charts of patients, which included sex, age, Eastern Cooperative Oncology Group Performance Status (ECOG PS), the site of primary tumor, histopathologic variant, pre-chemotherapy laboratory data, the number and distribution of visceral metastasis, and the first-line chemotherapy regimen. Patients treated with neoadjuvant chemotherapy $(\mathrm{n}=9)$, adjuvant chemotherapy ( $\mathrm{n}=$ $48)$, concurrent chemoradiotherapy $(n=3)$, and those who lack of pre-chemotherapy lymphocyte count and serum albumin levels for analysis $(\mathrm{n}=182)$ were excluded (Figure 1). All enrolled patients received systemic chemotherapy, and the choice of chemotherapy regimen was at the discretion of the physician. Standard radiological examinations, including computed tomography (CT) of abdomen and chest were used for both baseline tumor evaluation and the subsequent assessment of treatment response. The primary outcome of the study was OS, which was calculated from the date of metastatic disease confirmed to the date of death. Patients without disease progression or death were censored at the date of their last follow-up for survival analysis.

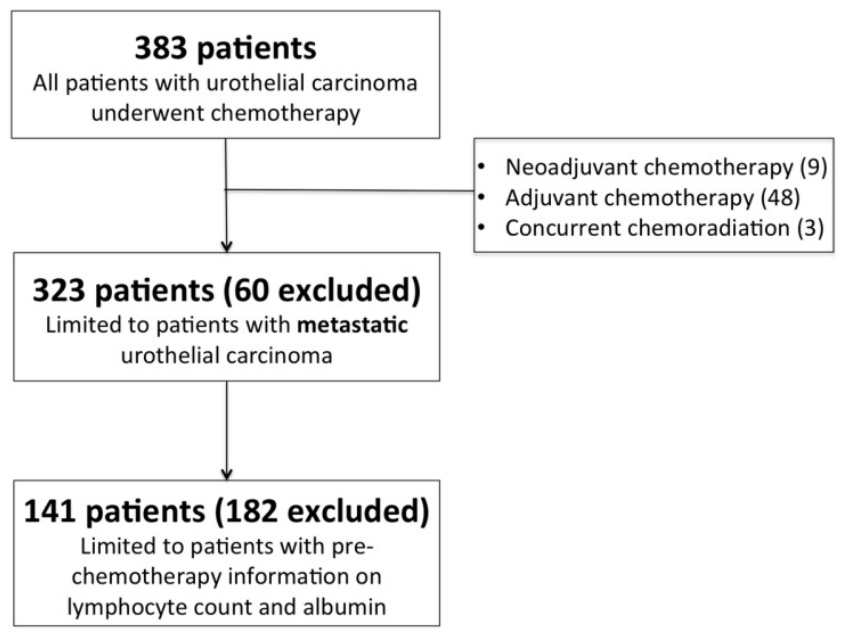

Figure 1. CONSORT flow diagram of patients.

\section{Laboratory measurements}

We obtained pre-chemotherapy blood parameters for all eligible patients within 1 week prior to first cycle of chemotherapy. Laboratory tests, including complete blood cell count (CBC) with differentials, lymphocyte count, and serum albumin were performed in local institute. The PNI was calculated according to the following formula: $10 \times$ serum albumin value $(\mathrm{g} / \mathrm{dL})+0.005 \times$ lymphocyte count (per $\left.\mathrm{mm}^{3}\right){ }^{14}$

\section{Statistical analysis}

We performed all statistical analyses by using SPSS 21.0 software (SPSS Inc., Chicago, IL, USA), and plotted survival figures using GraphPad Prism version 6.04 (GraphPad Software, La Jolla California, USA). The differences of categorical variables between two groups were compared by the Pearson chi-squared $\left(X^{2}\right)$ test or Fisher exact test. We used the receiver operating characteristic (ROC) curve analysis to obtain the optimal cut-off value of PNI for predictive overall survival. The estimated OS was calculated using the Kaplan-Meier method, and the statistical significance was compared using the log rank test. The independent prognostic factors for survival were identified in univariate and multivariate analyses using the Cox proportional hazards model. The hazard ratio (HR) with 95\% confidence interval $(\mathrm{CI})$ and $P$ value were calculated to quantify the strength of the association between the 
prognostic parameters and survival. All tests were two-sided, and $P<.05$ was considered statistically significant.

\section{Results}

\section{Patient characteristics}

The patient's demographics and clinicopathological characteristics are summarized in Table 1. Among the 141 patients enrolled in the study, 90 $(63.8 \%)$ were male. The median age at diagnosis was 64 years (interquartile range [IQR], 57-72). There were $82(58.2 \%)$ patients had upper urinary tract cancer and $54(38.3 \%)$ bladder cancer. Five $(3.5 \%)$ patients had multifocal primary sites. The majority of patients $(73.8 \%)$ had a good ECOG performance status (0-1), and more than half of patients $(52.5 \%)$ had visceral metastases. Of the 141 patients, $79(56 \%)$ had an impaired renal function ( $\mathrm{CCr}<60 \mathrm{~mL} / \mathrm{min}$ ), and 92 $(65.2 \%)$ patients received cisplatin-based chemotherapy.

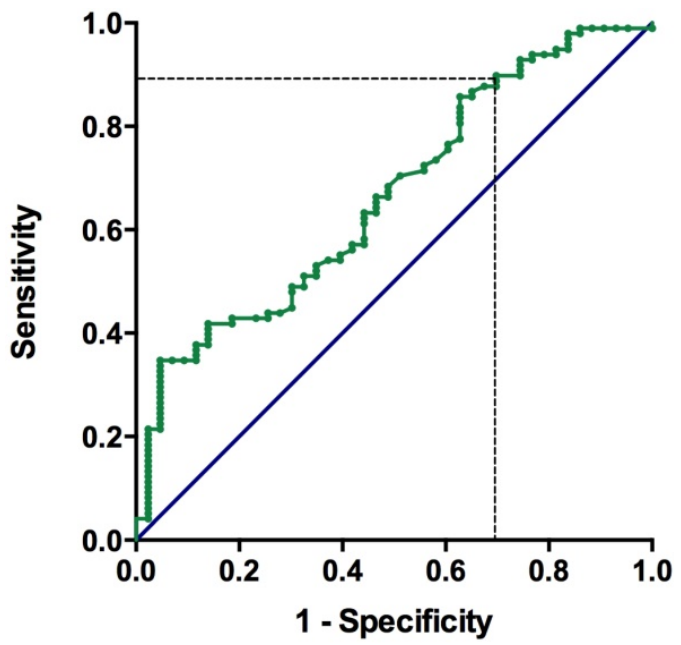

Figure 2. Receiver operating characteristic (ROC) curve for pre-chemotherapy $\mathrm{PNI}$ and survival in patients with metastatic urothelial carcinoma

\section{Association of PNI and overall survival}

Within the median follow-up period of 36.0 months (range: 27.6 - 44.4 months), 105 of 141 (74.5\%) patients died of disease. In general, the median OS were 13.5 months (95\% CI: 11.6 - 15.4 months). On univariate analysis, patients with PNI $<40$ had a significant shorter OS compared with those with PNI $>40$ (9.6 vs. 17.6 months, HR 2.41, 95\% CI 1.58 - 3.68, $\mathrm{p}<0.0001$; Figure 3). Table 2 listed the results of Kaplan Meier analysis of all demographic factors. Male (HR 1.56; 95\% CI 1.02-2.39; $\mathrm{p}=0.04), \mathrm{ECOG} \geq 2$ (HR 2.60; 95\% CI 1.62-4.15; $\mathrm{p}<0.0001$ ), presence of visceral metastasis (HR 2.55; 95\% CI 1.68-3.87; $\mathrm{p}<$ $0.0001)$ and $\mathrm{WBC} \geq 10,000 \times 10^{3} / \mu \mathrm{L}(\mathrm{HR} 1.52 ; 95 \% \mathrm{CI}$
1.00-2.30; $p=0.05$ ) were significantly associated with poor OS. To further validate the independent role of PNI, we performed Cox proportional regression analysis. We found that low PNI is an independent, negative prognostic factor for OS, yielding a significant increment in the mortality risk of $219 \%$ (HR 2.19; 95\% CI 1.40 - 3.43; $p=0.001$ ). Male (HR 1.93; 95\% CI 1.24 - 3.02; $\mathrm{p}=0.004)$, ECOG PS $\geq 2$ (HR 2.53; $95 \%$ CI $1.56-4.11 ; \mathrm{p}<0.0001)$, and presence of visceral metastasis (HR 1.90; 95\% CI $1.24-2.92 ; \mathrm{p}=$ 0.003 ) were also independent factors for OS.

Table 1. Clinical characteristics of advanced urothelial carcinoma patients grouped by PNI

\begin{tabular}{|c|c|c|c|c|}
\hline & All (n, \%) & $\mathrm{PNI}<40(\%)$ & $\mathrm{PNI} \geq 40(\%)$ & $P$ value \\
\hline Age (year) & & & & 0.81 \\
\hline$<65$ & $74(52.5)$ & $22(29.7)$ & $52(70.3)$ & \\
\hline$\geq 65$ & $67(47.5)$ & $18(27.9)$ & $49(73.1)$ & \\
\hline Gender & & & & 0.34 \\
\hline Female & $51(36.2)$ & $12(23.5)$ & $39(76.5)$ & \\
\hline Male & $90(63.8)$ & $29(32.2)$ & $61(67.8)$ & \\
\hline ECOG & & & & 0.40 \\
\hline $0-1$ & $104(73.8)$ & $28(26.9)$ & $76(73.1)$ & \\
\hline$\geq 2$ & $37(26.2)$ & $13(35.1)$ & $24(64.9)$ & \\
\hline Renal function (mL/min) & & & & 0.58 \\
\hline $\mathrm{CCr} \geq 60$ & $62(44.0)$ & $20(32.3)$ & $42(67.7)$ & \\
\hline $\mathrm{CCr}<60$ & $79(56.0)$ & $21(26.6)$ & $58(73.4)$ & \\
\hline Primary site & & & & 0.85 \\
\hline Upper tract & $82(58.2)$ & $25(30.5)$ & $57(69.5)$ & \\
\hline Bladder & $54(38.3)$ & $15(27.8)$ & $39(72.2)$ & \\
\hline Multifocal & $5(3.5)$ & $1(20)$ & $4(80)$ & \\
\hline Histopathologic variant & & & & 0.26 \\
\hline No & $89(63.1)$ & $29(32.6)$ & $60(67.4)$ & \\
\hline Yes & $52(36.9)$ & $12(23.1)$ & $40(76.9)$ & \\
\hline Visceral metastasis & & & & 0.009 \\
\hline No & $67(47.5)$ & $12(17.9)$ & $55(82.1)$ & \\
\hline Yes & $74(52.5)$ & $29(39.2)$ & $45(60.8)$ & \\
\hline Liver metastasis & & & & 0.054 \\
\hline No & $115(81.6)$ & $29(25.2)$ & $86(74.8)$ & \\
\hline Yes & $26(18.4)$ & $12(46.2)$ & $14(53.8)$ & \\
\hline Lung metastasis & & & & 0.16 \\
\hline No & $96(68.1)$ & $24(25.0)$ & $72(75.0)$ & \\
\hline Yes & 45 (31.9) & $17(37.8)$ & $28(62.2)$ & \\
\hline Bone metastasis & & & & 0.09 \\
\hline No & $116(82.3)$ & $30(25.9)$ & $86(74.1)$ & \\
\hline Yes & $25(17.7)$ & $11(44.0)$ & $14(56.0)$ & \\
\hline $\mathrm{WBC}\left(\times 10^{3} / \mu \mathrm{L}\right)$ & & & & 0.005 \\
\hline$<10$ & $97(68.8)$ & $21(21.6)$ & $76(78.4)$ & \\
\hline$\geq 10$ & $44(31.2)$ & $20(45.5)$ & $24(54.5)$ & \\
\hline NLR & & & & $<0.0001$ \\
\hline$<3$ & $55(39.0)$ & $3(5.5)$ & $52(94.5)$ & \\
\hline$\geq 3$ & $86(61.0)$ & $38(44.2)$ & $48(55.8)$ & \\
\hline Hemoglobin (g/dL) & & & & $<0.0001$ \\
\hline$\geq 10$ & $103(73.0)$ & 18 (17.5) & $85(82.5)$ & \\
\hline$<10$ & $38(27.0)$ & $23(60.5)$ & $15(39.5)$ & \\
\hline First-line chemotherapy & & & & 0.35 \\
\hline Cisplatin-based & $92(65.2)$ & $26(28.3)$ & $66(71.7)$ & \\
\hline Carboplatin-based & $36(25.5)$ & $13(36.1)$ & $23(63.9)$ & \\
\hline Other & $13(9.2)$ & $2(15.4)$ & $11(84.6)$ & \\
\hline
\end{tabular}


Table 1. Univariate and multivariate analysis of OS.

\begin{tabular}{|c|c|c|c|c|c|c|}
\hline \multirow[t]{3}{*}{ Characteristics } & \multicolumn{3}{|l|}{ PFS } & \multicolumn{3}{|l|}{ OS } \\
\hline & Univariate & Multivariate & & Univariate & Multivariate & \\
\hline & $p$ value & HR (95\% CI) & $p$ value & $p$ value & HR (95\% CI) & $p$ value \\
\hline $\begin{array}{l}\text { Age } \\
\geq 65 \text { vs. }<65\end{array}$ & 0.81 & $0.76(0.48-1.19)$ & 0.23 & 0.41 & $0.95(0.57-1.59)$ & 0.84 \\
\hline $\begin{array}{l}\text { Gender } \\
\text { Male vs. Female }\end{array}$ & 0.1 & $1.46(0.95-2.23)$ & 0.08 & 0.04 & 2.05 (1.24-3.37) & 0.005 \\
\hline $\begin{array}{l}\text { ECOG PS } \\
\geq 2 \text { vs. } 0-1\end{array}$ & 0.004 & $1.82(1.06-3.12)$ & 0.03 & $<0.0001$ & $2.69(1.48-4.91)$ & 0.001 \\
\hline $\begin{array}{l}\mathrm{CCr}(\mathrm{mL} / \mathrm{min}) \\
<60 \mathrm{vs} . \geq 60\end{array}$ & 0.31 & $0.74(0.45-1.23)$ & 0.24 & 0.4 & $0.74(0.40-1.25)$ & 0.27 \\
\hline $\begin{array}{l}\text { Primary site } \\
\text { Bladder vs. upper tract }\end{array}$ & 0.55 & $0.88(0.56-1.38)$ & 0.56 & 0.56 & $1.03(0.62-1.70)$ & 0.92 \\
\hline $\begin{array}{l}\text { Histologic variant } \\
\text { Yes vs. No }\end{array}$ & 0.33 & $1.56(1.00-2.42)$ & 0.05 & 0.57 & $1.47(0.88-2.43)$ & 0.14 \\
\hline $\begin{array}{l}\text { Visceral metastasis } \\
\text { Yes vs. No }\end{array}$ & $<0.0001$ & $1.90(1.22-2.98)$ & 0.005 & $<0.0001$ & $1.91(1.21-3.02)$ & 0.006 \\
\hline $\begin{array}{l}\mathrm{WBC}\left(\times 10^{3} / \mu \mathrm{L}\right) \\
\geq 10000 \mathrm{vs}<10000\end{array}$ & 0.08 & $1.37(0.85-2.19)$ & 0.19 & 0.05 & $1.44(0.85-2.44)$ & 0.18 \\
\hline $\begin{array}{l}\mathrm{Hb}(\mathrm{g} / \mathrm{dL}) \\
<10 \text { vs. } \geq 10\end{array}$ & 0.32 & $0.63(0.35-1.14)$ & 0.13 & 0.09 & $0.84(0.44-1.58)$ & 0.58 \\
\hline $\begin{array}{l}\text { PNI } \\
<40 \text { vs. } \geq 40\end{array}$ & 0.001 & $1.66(0.96-2.87)$ & 0.07 & $<0.0001$ & $2.28(1.31-3.96)$ & 0.003 \\
\hline $\begin{array}{l}1^{\text {st }} \text { line chemotherapy } \\
\text { Carboplatin vs. Cisplatin }\end{array}$ & 0.16 & $1.63(0.96-2.76)$ & 0.07 & 0.13 & $1.68(0.93-3.03)$ & 0.09 \\
\hline
\end{tabular}

Abbreviation: CCr, clearance of creatinine; CI, confidence interval; ECOG, Eastern Cooperative Oncology Group; HR, hazard ratio;

OS, overall survival; PFS, progression-free survival; PNI, prognostic nutritional index; PS, performance status; WBC, white blood cell count

\section{Determine the optimal cut-off value of PNI}

The median pre-treatment levels of albumin and lymphocyte count were $3.8 \mathrm{~g} / \mathrm{dL}(\mathrm{IQR}, 3.25$ - 4.1) and 1420 cells $/ \mu \mathrm{L}(\mathrm{IQR}, 942$ - 2024), respectively. The median value of PNI was 44.1 (25.6 - 62.1). The PNI values were significantly lower in patients with visceral metastasis $(p<0.0001)$, liver metastasis $(p=$ $0.05)$, lung metastasis $(\mathrm{p}=0.006)$, bone metastasis $(\mathrm{p}=$ $0.007)$, leukocytosis $(\mathrm{p}=0.006)$, and hemoglobin $(\mathrm{Hb})$ $<10 \mathrm{~g} / \mathrm{dL}$ ( $\mathrm{p}<0.0001$, shown as Table 1$)$. As shown in Figure 2, the area under the ROC curve for the PNI was 0.665 (95\% CI: 0.57 - 0.76). The optimal cut-off value of the PNI for predicting overall survival was 40.1 , with a sensitivity of $88.4 \%$ and a specificity of $37.8 \%$. We defined the recommended cut-off value of the PNI as 40.0 for further analysis.

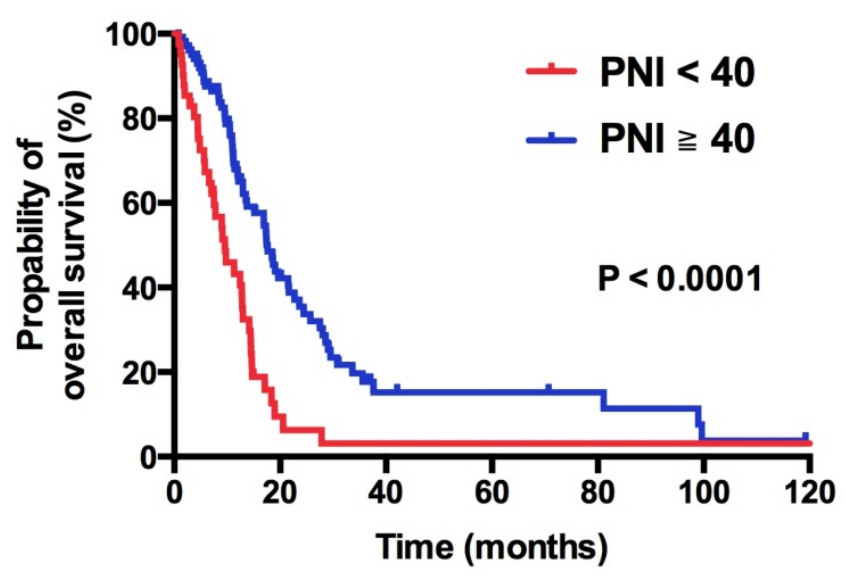

Figure 3. Kaplan-Meier overall survival curve stratified by PNI.

\section{Discussion}

To the best of our knowledge, this is the first study to evaluate the relationship between PNI and OS in patients with mUC. Patients with a low PNI were highly associated with visceral metastasis, leukocytosis and anemia. Furthermore, we found a low PNI value is an independent prognostic factor for OS in mUC patients treated with systemic chemotherapy. Thus, PNI is a clinically good tool for outcomes prediction and risk stratification in clinical studies, as well as patient counseling.

Recent evidence suggests that the nutritional and inflammatory status play an important role in the development and progression of cancer.[19] Herein, several prognostic markers had been developed for outcomes anticipation. The main models includes PNI,[15-18] NLR, [20, 21] platelet-to-lymphocyte ratio (PLR),[22] modified Glasgow prognostic scores (mGPS),[23] prognostic index (PI)[24] and Patient-Generated Subjective Global Assessment (PG-SGA).[25] PNI is calculated by serum albumin level and lymphocyte count, which reflects more comprehensively about nutritional and inflammatory status than other predictive models. For decades, previous studies have determined the survival impact of PNI in patients with various kinds of cancer. Geng et al. conducted a retrospective study of 321 patients with advanced pancreatic cancer and identified that a low PNI correlated significantly with a shorter OS and a higher level of TNF-a.[15] Ikeya et al. collected 80 patients with metastatic colorectal cancer treated with systemic chemotherapy and summarized that 
maintenance of PNI during pre- and postchemotherapy exhibited a better prognosis.[16] Du et al. reviewed 694 patients with non-disseminated nasopharyngeal cancer and reported that pre-therapy PNI and weight loss have significant predictive values for distant metastasis and mortality.[17] Sheng et al. analyzed 144 non-small cell lung cancer treated with targeted therapy and suggested a low PNI was correlated with worse outcomes.[18] Our study confirmed the independent prognostic role of PNI in mUC patients, and the results were highly consistent with previously published.

The pathophysiology of nutritional and inflammatory response affecting survival is complicated. It is well known that malnutrition usually causes humeral and cellular immune dysfunction, inflammatory response alterations, and delay of the wound healing process.[26] Sonpavde et al. recently proved that albumin, which reflects the nutritional status, was externally validated as a prognostic factor for overall survival in patients with advanced urothelial carcinoma receiving salvage systemic chemotherapy.[27] Furthermore, several studies also confirmed the associations between clinical outcomes systemic inflammation in patients with various cancer types. A recent comprehensive review proposed that cancer-related inflammation is the seventh hallmark of cancer.[28] Inflammation may induce cancer microenvironment change and increase vascular permeability that are in favor of cancer progression.[29] One of these inflammatory signals is the extracellular matrix component versican, which leads to macrophage activation and production of the metastasis-promoting cytokine TNF-a.[30] Meanwhile, a low lymphocyte count is associated with impaired host cell-mediated immunity since lymphocytes have the pivotal role in cytotoxic cell death and cytokine production that inhibits proliferation and metastatic activity of tumor cells.[31] Taken together, both nutritional and systemic inflammatory response are crucial in cancer development and metastasis, and also essential in estimating patient's prognosis. Further specific targeting therapy on cancer-associated inflammatory process deems to be a brand new milestone of cancer treatment.

The optimal value of PNI to predict prognosis in patients with cancer remains undetermined. Preceding literatures have reported various cutoff values in their studies. Migita et al analyzed 548 patients with gastric cancer who underwent gastrectomy and set PNI at 48 because the sensitivity and specificity for the 5-year OS were $82.3 \%$ and 57.9 $\%$, respectively.[32] Jiang et al also summarized 386 elderly patients with gastric cancer and set their cut-off value at 46 with close correlation between PNI and age.[26] Sun et al conducted a meta-analysis to determine the predictive significance of PNI in cancer and found the cutoff value of PNI was usually set at 45 , because a PNI $<45$ is defined as moderate to severe malnutrition.[33] However, the cut-off value of PNI in this present study was determined to be at 40 according to ROC curve analysis. When PNI was chosen at 40 , the sensitivity and specificity were $88.4 \%$ and $37.8 \%$, respectively. Moreover, we also investigate the differences in the clinicopathologic factors and survival between PNI-high and PNI-low groups in patients with mUC. Based on our study, patients in PNI-low group had more visceral metastasis, leukocytosis, anemia and high NLR. These were all known as poor prognostic factors for $\mathrm{mUC}$, resulting in worse outcomes of patients in PNI-low group. Also, the multivariable analysis confirmed the predictive role of PNI in patients with mUC. After adjusting with possible confounding factors, PNI is an independently significant viable that affecting the survival of patients with mUC.

Nonetheless, there are several potential limitations in our study. First, this is a retrospective analysis in a single institution with a relatively small sample size, which may limit the power of our study. Second, PNI was calculated by CRP and lymphocyte counts. It is easily interfered by external environment, such as infection, stress and local inflammation except tumors. Meanwhile, the timing of PNI assessment was not consistent. Given that our study was conducted by chart review, we are unable to realize the actual condition of each patient. This might be a major bias of our study. Third, the characteristics of patients were uncontrolled and heterogeneous between PNI-high and PNI-low groups. These imbalanced baselines have some influences on the OS of our patients. Therefore, a prospective, multi-institutional, well-controlled study is warranted to validate our results.

\section{Conclusions}

In conclusion, this study disclosed that PNI is an independent prognostic factor for patients with $\mathrm{mUC}$ treated with chemotherapy. Based on our result, routine assessment of PNI is suggested and crucial. Our work is clinically useful for anticipation of outcomes, risks stratification in clinical studies as well as patients counseling.

\section{Acknowledgement}

We thank the multidisciplinary team of genitourology cancer at our institute for their gracious assistance and cooperation. 


\section{Competing Interests}

The authors have declared that no competing interest exists.

\section{References}

1. Siegel R, Naishadham D, Jemal A. Cancer statistics, 2013. CA Cancer J Clin 2013; 63: 11-30.

2. Pliarchopoulou K, Laschos K, Pectasides D. Current chemotherapeutic options for the treatment of advanced bladder cancer: a review. Urol Oncol 2013; 31: 294-302.

3. Witjes JA, Comperat E, Cowan NC, et al. EAU guidelines on muscle-invasive and metastatic bladder cancer: summary of the 2013 guidelines. Eur Urol 2014; 65: 778-92.

4. Booth CM, Siemens DR, Li G, et al. Perioperative chemotherapy for muscle-invasive bladder cancer: A population-based outcomes study. Cancer 2014; 120: 1630-8.

5. Apolo AB, Ostrovnaya I, Halabi S, et al. Prognostic model for predicting survival of patients with metastatic urothelial cancer treated with cisplatin-based chemotherapy. J Natl Cancer Inst 2013; 105: 499-503.

6. Tanaka N, Kikuchi E, Kanao K, et al. Patient characteristics and outcomes in metastatic upper tract urothelial carcinoma after radical nephroureterectomy: the experience of Japanese multi-institutions. BJU Int 2013; 112: E28-34.

7. Xylinas E, Rink M, Margulis V, et al. Impact of renal function on eligibility for chemotherapy and survival in patients who have undergone radical nephro-ureterectomy. BJU Int 2013; 112: 453-61.

8. Galsky M, Moshier E, Krege S, et al. Nomogram for predicting survival in patients with unresectable and/or metastatic urothelial cancer who are treated with cisplatin-based chemotherapy. Cancer 2013; 119: 3012-19.

9. Hsieh MC, Sung MT, Chiang PH, et al. The Prognostic Impact of Histopathological Variants in Patients with Advanced Urothelial Carcinoma. Plos One 2015; 10: e0129268.

10. Iseki Y, Shibutani M, Maeda K, et al. Impact of the Preoperative Controlling Nutritional Status (CONUT) Score on the Survival after Curative Surgery for Colorectal Cancer. PLoS One 2015; 10: e0132488.

11. Tan CS, Read JA, Phan VH, et al. The relationship between nutritional status, inflammatory markers and survival in patients with advanced cancer: a prospective cohort study. Support Care Cancer 2015; 23: 385-91.

12. Kinoshita A, Onoda H, Imai N, et al. The C-reactive protein/albumin ratio, a novel inflammation-based prognostic score, predicts outcomes in patients with hepatocellular carcinoma. Ann Surg Oncol 2015; 22: 803-10.

13. Sun KY, Xu JB, Chen SL, et al. Novel immunological and nutritional-based prognostic index for gastric cancer. World J Gastroenterol 2015; 21: 5961-71.

14. Onodera T, et al. Prognostic nutritional index in gastrointestinal surgery of malnourished cancer patients (in Japanese with English abstract). Nihon Geka Gakkai Zasshi 1984; 85: 1001-5.

15. Geng Y, Qi Q, Sun M, et al. Prognostic nutritional index predicts survival and correlates with systemic inflammatory response in advanced pancreatic cancer. Eur J Surg Oncol 2015; 41: 1508-14.

16. Ikeya $T$, Shibutani $M$, Maeda $K$, et al. Maintenance of the nutritional prognostic index predicts survival in patients with unresectable metastatic colorectal cancer. J Cancer Res Clin Oncol 2015; 141: 307-13.

17. Du XJ, Tang LL, Mao YP, et al. Value of the prognostic nutritional index and weight loss in predicting metastasis and long-term mortality in nasopharyngeal carcinoma. J Transl Med 2015; 13: 364.

18. Sheng J, Yang YP, Ma YX, et al. Low Prognostic Nutritional Index Correlates with Worse Survival in Patients with Advanced NSCLC following EGFR-TKIs. PLoS One 2016; 11: e0147226.

19. Grivennikov SI, Greten FR, Karin M. Immunity, inflammation, and cancer. Cell 2010; 140: 883-99.

20. Taguchi S, Nakagawa T, Matsumoto A, et al. Pretreatment neutrophil-to-lymphocyte ratio as an independent predictor of survival in patients with metastatic urothelial carcinoma: A multi-institutional study. Int J Urol 2015; 22: 638-43.

21. Luo Y, She DL, Xiong H, et al. Pretreatment Neutrophil to Lymphocyte Ratio as a Prognostic Predictor of Urologic Tumors: A Systematic Review and Meta-Analysis. Medicine (Baltimore) 2015; 94: e1670.

22. Fan W, Zhang Y, Wang $Y$, et al. Neutrophil-to-lymphocyte and platelet-to-lymphocyte ratios as predictors of survival and metastasis for recurrent hepatocellular carcinoma after transarterial chemoembolization. PLoS One 2015; 10: e0119312.

23. Ferro M, De Cobelli O, Buonerba C, et al. Modified Glasgow Prognostic Score is Associated With Risk of Recurrence in Bladder Cancer Patients After Radical Cystectomy: A Multicenter Experience. Medicine (Baltimore) 2015; 94: e1861.

24. Maeda K, Shibutani M, Otani H, et al. Low nutritional prognostic index correlates with poor survival in patients with stage IV colorectal cancer following palliative resection of the primary tumor. World J Surg 2014; 38: 1217-22.

25. Gioulbasanis I, Martin L, Baracos VE, et al. Nutritional assessment in overweight and obese patients with metastatic cancer: does it make sense? Ann Oncol 2015; 26: 217-21.
26. Jiang N, Deng JY, Ding XW, et al. Prognostic nutritional index predicts postoperative complications and long-term outcomes of gastric cancer. World J Gastroenterol 2014; 20: 10537-44.

27. Sonpavde G, Pond GR, Rosenberg JE, et al. Improved 5-Factor Prognostic Classification of Patients Receiving Salvage Systemic Therapy for Advanced Urothelial Carcinoma. J Urol 2016; 195: 277-82.

28. Colotta F, Allavena P, Sica A, et al. Cancer-related inflammation, the seventh hallmark of cancer:links to genetic instability. Carcinogenesis 2009; 30: 1073-81.

29. Rossi L, Santoni M, Crabb SJ, et al. High neutrophil-to-lymphocyte ratio persistent during first-line chemotherapy predicts poor clinical outcome in patients with advanced urothelial cancer. Ann Surg Oncol 2015; 22: 1377-84.

30. Kim S, Takahashi H, Lin WW, et al. Carcinoma-produced factors activate myeloid cells through TLR2 to stimulate metastasis. Nature 2009; 457: 102-6.

31. Ding PR, An X, Zhang RX, et al. Elevated preoperative neutrophil to lymphocyte ratio predicts risk of recurrence following curative resection for stage IIA colon cancer. Int J Colorectal Dis 2010; 25: 1427-33.

32. Migita K, Takayama T, Saeki K, et al. The prognostic nutritional index predicts long-term outcomes of gastric cancer patients independent of tumor stage. Ann Surg Oncol 2013; 20: 2647-54.

33. Sun $\mathrm{K}$, Chen $\mathrm{S}, \mathrm{Xu} \mathrm{J}$, et al. The prognostic significance of the prognostic nutritional index in cancer: a systematic review and meta-analysis. J Cancer Res Clin Oncol 2014; 140: 1537-49. 